\title{
VIT 030 - THE SPECIAL CODE FOR COMPUTER SIMULATION OF THE RF PROCESS IN VEPP-5 PREINJECTOR.
}

\author{
V.V.Podlevskih \\ Budker INP, 630090 Novosibirsk, Russia
}

\begin{abstract}
This paper presents the analycis of algoritm and description of the special code for computer simulation of the RF process in linear accelerator.

This program may be used for preliminary analysis of the RF process, RF system setup and monitoring.

\section{INTRODUCTION}

RF system of VEPP-5 preinjector consists of the separate RF module. The main elements of each module are:

a) klystron amplifier;

b) SLED type power multiplying system consisting of two high Q cavities and 3dB directional coupler;

c) accelerating section connected with waveguide and RF load by a coupler;

d) RF load;

f) waveguide section including straight waveguide parts, $\mathrm{E}$ and $\mathrm{H}$ turns, directional coupler etc.

The objectives of RF process simulation are:

a) representation of each RF system element by a limited set of parameters mostly characterizing the operation of the device;

b) creation of a model of interaction between separate elements of the RF system.

RF process under investigation (that can be measured) are electromagnetic wave amplitudes in the defined cross-sections of the waveguide section (including the disk loaded accelerating structure).
\end{abstract}

\section{BASIC EQUATION}

Scheme of the RF modules of VEPP-5 preinjector RF system module is shown in Fig.1.
A spectral representation of RF process is their most suitable form for the points, marked in Fig1. Time depend and spectral representation are connected by the following equations:

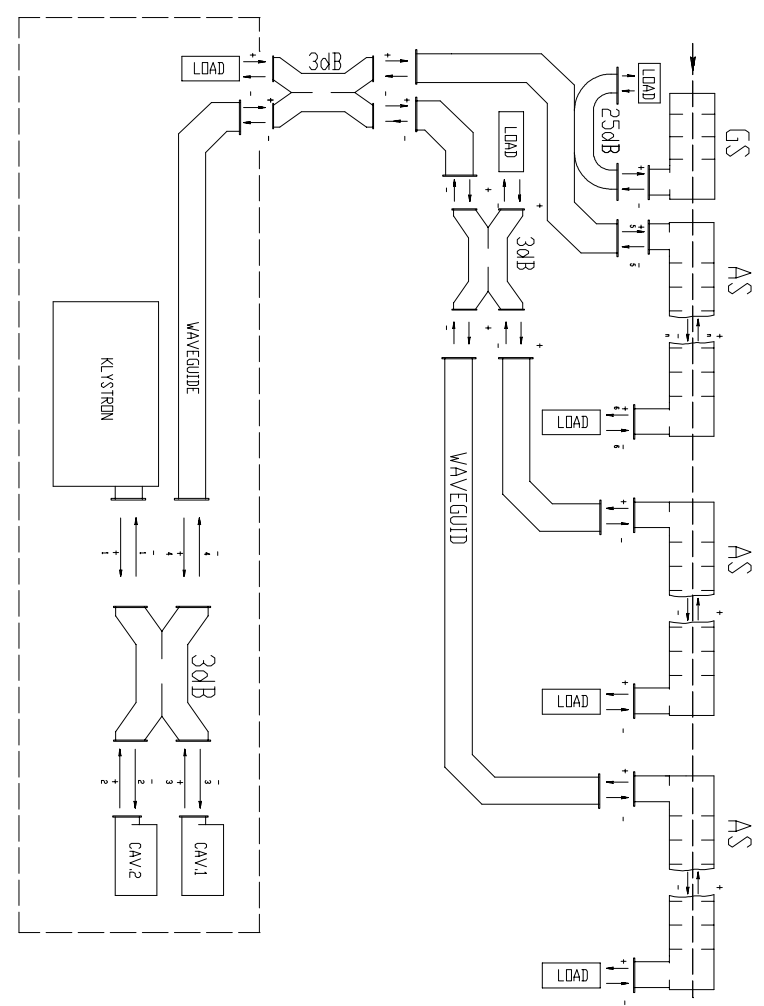

Figure 1: Scheme of the RF module of VEPP-5 preinjector.

$$
\begin{aligned}
& V(f)=\frac{1}{\sqrt{2 \pi}} \int_{-\infty}^{\infty} U(t) e^{-i 2 \pi f t} d t \\
& U(t)=\frac{1}{\sqrt{2 \pi}} \int_{-\infty}^{\infty} U(f) e^{i 2 \pi f t} d t
\end{aligned}
$$


In terms of Fig.1 designations connection between the signal ganged by $3 \mathrm{~dB}$ directional coupler has the following form:

$$
\begin{aligned}
& \left(\begin{array}{l}
V_{1}^{-} \\
V_{2}^{-} \\
V_{3}^{-} \\
V_{4}^{-}
\end{array}\right)=\frac{1}{\sqrt{2}}\left(\begin{array}{cccc}
0 & 1 & -i & 0 \\
1 & 0 & 0 & -i \\
-i & 0 & 0 & 1 \\
0 & -i & 1 & 0
\end{array}\right)\left(\begin{array}{c}
V_{1}^{+} \\
V_{2}^{+} \\
V_{3}^{+} \\
V_{4}^{+}
\end{array}\right) \\
& \left(\begin{array}{l}
V_{1}^{+} \\
V_{2}^{+} \\
V_{3}^{+} \\
V_{4}^{+}
\end{array}\right)=\frac{1}{\sqrt{2}}\left(\begin{array}{cccc}
k_{1} & 0 & 0 & 0 \\
0 & k_{2} & 0 & 0 \\
0 & 0 & k_{3} & 0 \\
0 & 0 & 0 & k_{4}
\end{array}\right)\left(\begin{array}{l}
V_{1}^{+} \\
V_{2}^{+} \\
V_{3}^{+} \\
V_{4}^{+}
\end{array}\right)+\left(\begin{array}{l}
V_{0} \\
0 \\
0 \\
0
\end{array}\right)
\end{aligned}
$$

Where $V_{0}$ is an amplitude of the wave at the klystron amplifier output when operating to the matched load.

All the variables and constants in equations $(3,4)$ are functions of a frequency (i.e. $V_{i}^{ \pm} \equiv V_{i}^{ \pm}(f)$ and $\left.k_{i}^{ \pm} \equiv k_{i}^{ \pm}(f)\right)$. A compact matrix form of the equations 3 and 4 is: $V^{-}=S V^{+}$and $V^{-}=K_{S} V^{-}+V_{0}$ with joint solution: $V^{-}=\left(S^{-1}-K_{S}\right)^{-1} V_{0}$ and $V^{+}=S^{-1} V^{-}$.

The following equations connect wave amplitudes at the input and output of the accelerating structure:

The following equation describes connection between signals at the input and output of the waveguide section which connects the power multiplying system with the accelerating section:

$$
\left(\begin{array}{c}
V_{4}^{+} \\
V_{5}^{+}
\end{array}\right)=\left(\begin{array}{ll}
k_{44} & k_{45} \\
k_{54} & k_{55}
\end{array}\right)\left(\begin{array}{l}
V_{4}^{-} \\
V_{5}^{-}
\end{array}\right)
$$

or in matrix form: $V^{+}=K_{L} V^{-}$, where $K_{L}$ is $S$ matrix of the waveguide.

The following equations connect wave amplitudes at the input and output of the accelerating structure:

$$
\left(\begin{array}{c}
V_{5}^{-} \\
V_{6}^{-}
\end{array}\right)=\left(\begin{array}{ll}
k_{55} & k_{56} \\
k_{65} & k_{66}
\end{array}\right)\left(\begin{array}{c}
V_{5}^{+} \\
V_{6}^{+}
\end{array}\right)
$$

or in matrix form: $V^{-}=K_{a s} V^{+}$, the matrix $K_{a s}$ describes in following section.

It may be shown that Eqs. $(4,5,6)$ can be united in one (that is the reason for there common indexation), salving which sumultaniosly with Eqs.(3) determines uniquely all components of vectors:

$V^{-}=\left(V_{1}^{-}, V_{2}^{-}, V_{3}^{-}, V_{4}^{-}, V_{5}^{-}, V_{6}^{-}\right) V^{+}=\left(V_{1}^{+}, V_{2}^{+}, V_{3}^{+}, V_{4}^{+}, V_{5}^{+}, V_{6}^{+}\right)$ at given input pulse $V_{0}$ (f) and matrix coefficient $K_{L}(f)$ and $K_{a s}(f)$.

\section{SIMULATION OF RF ELEMENTS}

\subsection{Klystron amplifier.}

Klystron amplifier is an RF power source for each module in preinjector. If an output signal of the klystron amplifier $U_{1}^{+}(t)$ does not change occasionally from pulse to pulse, it may be represented as a sum:

$$
U_{1}^{+}=U_{0}(t)+\int_{0}^{t} K^{k l}\left(t, t^{\prime}\right) U_{1}^{-}\left(t^{\prime}\right) d t^{\prime}
$$

where $U_{0}(t)$ is an output signal of the klystron amplifier operating to the matched load. The addend determines the klystron output signal depending on possible reflections from the RF module waveguide section. If $K^{k l}\left(t, t^{\prime}\right) \equiv K^{k l}\left(t-t^{\prime}\right)$, equation (7) becomes:

$$
U_{1}^{+}(t)=U_{0}(t)+\int_{0}^{t} K^{k l}\left(t-t^{\prime}\right) U_{1}^{-}\left(t^{\prime}\right) d t^{\prime}
$$

or in spectral representation:

$$
V_{1}^{+}(f)=V_{0}(f)+K^{k l}(f) V_{1}^{-}(f)
$$

According to designations introduced in section 1 coefficient $k_{1}$ in (2) is equal to coefficient $K^{k l}$ in (9)

\subsection{Power multiplying system cavities.}

Power multiplying system contains (see Fig.1) two hight-Q cavities coupled by a slot with the input waveguide. The general view of each cavity as well as circuit representation are shown in Fig.2.
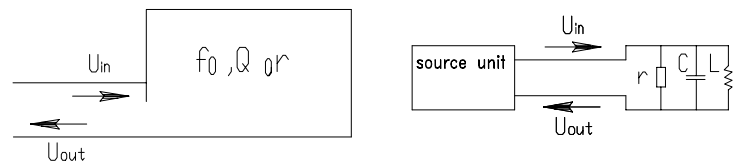

Figure 2: RF cavity coupled with input waveguide and its circuit representation. is:

Coupling between the input and output cavity signals

$$
V_{\text {out }}(f)=K^{s l}(f) V_{\text {in }}(f)
$$

where $K^{s l}(f)$ is frequency dependence of the cavity reflection coefficient:

$$
K^{s l}(f)=\frac{r-1-i \xi}{r+1+i \xi}
$$


$r$ is the coupling coefficient between the cavity and input waveguide;

$\xi=Q_{0} \frac{\left(f-f_{0}\right)}{f_{0}}$ is the generalized cavity frequency detuning;

$f_{0}, Q_{0}$ are the cavity resonant frequency and $Q$ factor.

According to designations introduced in section 1, coefficient $k_{22}$ and $k_{33}$ in Eqs. $(1,2)$ are equal to the reflection coefficient determined by (11) with substituting in it parameters of each cavity.

\subsection{Accelerating structure.}

The accelerating structure is designed as a round diskloaded waveguide and is connected with RF power source and RF load through the input and output couplers. Equivalent circuit of the accelerating structure is shown in Fig.3.

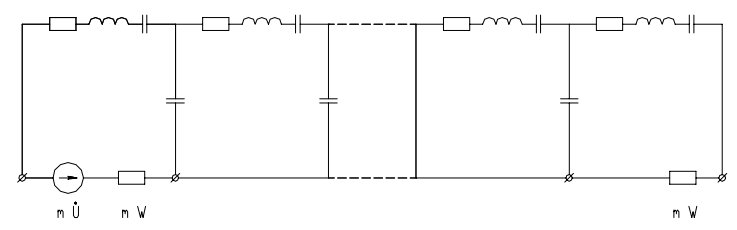

Figure 3: Circuit representation of the accelerating structure.

In this case we can write:

$$
\begin{aligned}
& \left(\begin{array}{ccccccc}
a_{1} & \frac{k_{1}}{2} & 0 & 0 & \cdot & \cdot & \cdot \\
\frac{k_{1}}{2} & a_{2} & \frac{k_{2}}{2} & 0 & \cdot & \cdot & \cdot \\
\cdot & \frac{k_{2}}{2} & a_{3} & \frac{k_{3}}{2} & 0 & \cdot & \cdot \\
\cdot & \cdot & \cdot & \cdot & \cdot & \cdot & \cdot \\
0 & 0 & 0 & 0 & 0 & \frac{k_{N-1}}{2} & a_{N}
\end{array}\right)\left(\begin{array}{l}
X_{1} \\
X_{2} \\
X_{3} \\
0 \\
0 \\
0 \\
X_{N}
\end{array}\right)=\left(\begin{array}{l}
V_{0} \\
0 \\
0 \\
0 \\
0 \\
0 \\
0
\end{array}\right)(12) \\
& a_{1}=1-\frac{f^{2}}{f_{1}^{2}}+i \frac{f}{f_{1}} \frac{1+\beta_{1}}{Q_{1}}, \mathrm{a}_{\mathrm{N}}=1-\frac{f^{2}}{f_{N}^{2}}+i \frac{f}{f_{N}} \frac{1+\beta_{N}}{Q_{N}} \\
& \text { and } a_{n}=1-\frac{f^{2}}{f_{n}^{2}}+i \frac{f}{f_{n}} \frac{1}{Q_{n}}, \text { where: }
\end{aligned}
$$

$f_{n}, Q_{n}$ are the resonant frequency and Q-factor of the n-th cell;

$\beta_{1}, \beta_{N}$ are the coupling coefficient between 1-th and

$\mathrm{N}$-th cavities with input and output waveguide.

$k_{n}$ is the coupling coefficient $\mathrm{n}$-th and $(\mathrm{n}+1)$-th cell.

The reflection coefficient of the accelerating structure and the transmittient coefficient for $\mathrm{n}$-th cell may be found directly from the matrix coefficient of the system (12) :

$$
\begin{aligned}
& k_{55}=-1-2 i \frac{f_{1}}{f} \frac{\beta_{1}}{Q_{1}} \frac{\Delta_{1}}{\Delta} \\
& k_{5 n}=\frac{\Delta_{1}}{\Delta}
\end{aligned}
$$

where $\Delta$ is the determinant of the system (12), $\Delta_{n}$ is the determinant of the system (12) without $\mathrm{n}$-th column and nth line.

\section{CONCLUSION}

The mathematical algorithm described above was completely realized in the original VIT 030 code. It allows us to solve the linear accelerator RF module design problems as well as questions arising during RF module tests and conditioning fast and with good quality.

The code which will allow us to take into account discharges in the system and to perform the expeditious diagnostics of the RF module is under development now on the base of VIT 030 code.

\section{REFERENCES}

[1] Simon Ramo, John R., "Field and waves in communication electronics", Second Edition, John Willey \& Sons, 1984.

[2] R.E. Collin, "Foundations for microwave engineering", Mc GrawHill, 1955. 